\title{
EMOCIONES EN LA CONDUCCIÓN DE VEHÍCULOS: DIFERENCIAS ENTRE ESTUDIANTES Y PROFESIONALES
}

\author{
J.L. Antoñanzas \\ Universidad de Zaragoza \\ jlantona@unizar.es \\ A.Lope \\ Universidad de Zaragoza \\ C. Salavera \\ Universidad de Zaragoza \\ C. Bericat \\ Universidad de Zaragoza
}

Fecha de Recepción: 5 Agosto 2019 Fecha de Admisión: 25 Septiembre 2019

\section{RESUMEN}

La conducción supone la puesta en práctica de toda una serie de emociones y motivaciones. Un sujeto a los mandos de un vehículo experimenta diversos estados emocionales y motivacionales que le van a influir de forma directa en su toma de decisiones. La ansiedad ha sido una de las emociones más estudiada a lo largo de los años como variable que influye en la conducción. Una buena conducción requiere que asumamos la tarea infinita de prevenir errores y suprimir decisiones irracionales con lo que ello conlleva de emociones. Aquellos conductores que poseen una mayor capacidad de control y regulación de sus emociones, a su vez controlan mejor los niveles de ansiedad

Palabras clave: emociones; conducción; estudiantes; profesionales.

\section{ABSTRACT}

Emotions in driving vehicles: differences between students and professionals. The driving supposed the implementation of a whole series of emotions and motivations. A subject to the controls of a vehicle of experimentation various emotional and motivational states that will influence him directly in his decision making. Anxiety has been one of the most studied emotions over the years as a variable that influences driving. Good driving requires that we assume the infinite task of preventing errors and suppressing irrational decisions with what this entails with emotions. Those drivers who have a greater ability to control and regulate their emotions, in turn control anxiety levels better

Keywords: emotions; driving; students; professionals. 


\section{EMOCIONES EN LA CONDUCCIÓN DE VEHÍCULOS: DIFERENCIAS ENTRE ESTUDIANTES Y PROFESIONALES}

\section{OBJETIVOS}

La presente investigación trato de detectar las emociones que se producen en la conducción de vehículos. Se evaluaron las capacidades emocionales de los conductores, así como los niveles de ansiedad que estos experimentan cuando están en la tarea de llevar un vehículo.

\section{INTRODUCCIÓN}

\section{Emoción y conducción}

Una buena conducción requiere que asumamos la tarea infinita de prevenir errores y suprimir decisiones irracionales. Ya que las emociones son fuente de juicios irracionales y de costosos errores, se requiere la educación de nuestras emociones de tráfico y, por tanto, su estudio.

Las habilidades afectivas de los conductores establecen las actitudes que se derivan del sistema motivacional y el socioemocional. Las emociones en el tráfico gobiernan nuestra competitividad y agresividad, así como nuestra calma, optimismo y compasión. Nuestro pensamiento se deriva de nuestras actitudes y motivos. Ya que pensamos conforme a cómo sentimos, los sentimientos negativos promueven pensamientos pesimistas. Nuestras acciones son las consecuencias de las actitudes que mantenemos y los pensamientos que tenemos.

Las emociones en el tráfico pueden tener un carácter negativo (enfado), pero también un carácter positivo (preocupación por otros usuarios de la vía). En este sentido, incluso sin darse cuenta, se puede insultar a otro usuario de la vía, conducir más rápido porque resulta divertido, olvidarse de las rutinas habituales de modo impulsivo o despreocuparse de otros usuarios vulnerables de la vía (Levelt, 2003).

Las emociones en el escenario vial no están causadas solamente por eventos relacionados con el tráfico. El modo en que uno se siente antes de iniciar un trayecto es tan importante como el estado emocional durante el curso de su participación en el tráfico (Swov, 2005).

Las emociones en todo lo relacionado con aspectos internos son difíciles de controlar, no así en sus aspectos externos. En general, en las emociones se generan dos problemas, principalmente, el conflicto de predominio entre ellos y la discordancia entre ellos y la razón.

Hoy en día, podemos afirmar que la relación entre emoción y conducción está más que demostrada. Son varios los estudios que ponen en relación a la emoción y a los comportamientos en el tráfico (Bisquerra, 2000; Cale \& Shaked, 2004; Clapp et al., 2011; Stephens \& Groeger, 2009; Swov, 2003; Vaa, 2004).

La mayor parte de las emociones que experimentamos en el vehículo son consecuencia de las emociones generadas en todos los ámbitos de la vida, así como por la propia conducción. Dichas emociones son anteriores al razonamiento y, por ende, afectan al procesamiento, las actitudes y la ejecución.

Prueba de ello es que tanto la propia Administración como las agencias de publicidad la utilizan como estrategia para modificar dichos procesos. Ahora bien, las emociones pueden provocar que nuestro comportamiento sea más seguro o bien pueda estar guiado hacia el riesgo.

Las emociones influyen en la conducción a través de la personalidad del sujeto, bien sea a través de la autoestima, la autonomía, etc., pero también por acontecimientos puntuales en un día, situaciones familiares, económicas o laborales etc., es decir, por situaciones que provocan ciertos comportamientos arriesgados y que además se suman a la propia actividad de conducir.

Una de las cuestiones fundamentales en relación con la conducción es que las propias emociones se pueden y se deben educar: bien sea a través del ámbito familiar y escolar o bien con los grupos de iguales, en el propio entorno laboral, las academias de conductores, y/o en los profesiona- 
les del transporte. En todo este contexto hay que subrayar que existe una incongruencia entre los mensajes procedentes de los medios de comunicación y la educación de las emociones hacia la seguridad.

Por ello, se debe incrementar la inteligencia emocional con un mejor conocimiento de lo que es, con una mayor comprensión de lo que significa, intentando tener un mayor control, regulación, expresión y afrontamiento de las propias emociones. Ello supondrá un incremento de autoestima y autoconfianza además de servir para detectar las emociones del resto de los conductores (Alonso et al., 2006a).

\section{Ansiedad y conducción}

La tarea de conducir un vehículo conlleva la presencia de ciertos estresores de mayor o menor intensidad. Dentro de ellos, el estresor que más se ha estudiado por su importancia y por su relación directa con la conducción ha sido la congestión de tráfico. Estas circunstancias son potencialmente estresantes debido a los retrasos y a la hostilidad que algunas veces provocan (Turner, Layton \& Simons, 1975). Un estudio realizado por Novaco, Stokols, Campbell \& Stokols (1979) ponía de manifiesto que las personas que viajan en automóvil por aquellas rutas con mayor volumen de tráfico, en comparación con los que viajan por rutas más fluidas, muestran mayores alteraciones fisiológicas (elevaciones en la presión sanguínea, reducciones en la destreza motora, etc.). Estudios posteriores, también encuentran en los conductores expuestos a grandes congestiones de tráfico, elevaciones en la presión sanguínea y déficits en la realización de respuestas (Schaeffer, 2000).

La mayor parte de estos estudios se ha dedicado a la evaluación de las respuestas fisiológicas de los conductores. Es indudable que la exposición a determinadas situaciones de tráfico está relacionada con la actividad del sistema nerviosos simpático.

También, es cierto que no toda respuesta fisiológica puede ser considerada como un índice de la actividad del sistema nervioso. Sí que existe, por otra parte, cierta consistencia de los sujetos a varias medidas fisiológicas, la conductancia de la piel y la tasa cardiaca parecen ser buenas medidas del estado de ansiedad del sujeto. En este sentido, una investigación llevada a cabo por Robertson (1987), en la que aplica el Driver Behavior Inventory (Gulian, Glendon, Matthews, Davies \& Debney, 1988), encuentra concomitancia entre dos variables del inventario: alerta en la conducción y frustración y tensión al no poder adelantar, y aceleración de la tasa cardiaca en sujetos conductores de automóviles.

El Driver Behavior Inventory intenta dar una visión global de todas las dimensiones y factores que contribuyen a la aparición y el mantenimiento del estrés en la conducción. Dicho modelo está basado en el modelo transaccional del estrés de Lazarus (1960). El estrés aparece cuando la percepción de las demandas de la tarea excede de las habilidades del conductor para enfrentarse a ellas. Se le exige al sujeto determinados esfuerzos durante la conducción para controlar el estrés. En este sentido, hay que recalcar que no todos los individuos perciben de la misma forma las diferentes situaciones del tráfico. Esas diferencias individuales marcan los comportamientos de los conductores, es un modelo que enfatiza las diferentes valoraciones cognitivas que se realizan de los acontecimientos como determinantes en el nivel de respuesta de estrés.

Desde esta perspectiva, se entiende el estrés del conductor como un complejo de respuestas que realiza el sujeto en la conducción. En dichas respuestas intervienen tanto factores personales como situacionales. Puede ser una reacción emocional (la ansiedad), fisiológica (aumento de la tasa cardiaca) y/o comportamental (conducción agresiva). Así, el modelo considera que el estrés puede experimentarse en dos niveles distintos:

En un primer nivel, el estrés está determinado por situaciones muy concretas que se presentan 


\section{EMOCIONES EN LA CONDUCCIÓN DE VEHÍCULOS: DIFERENCIAS ENTRE ESTUDIANTES Y PROFESIONALES}

ocasionalmente en el tráfico y sobre las cuales el sujeto tiene un control muy limitado. El nivel más alto de ansiedad en tales situaciones actuaria impidiendo que el sujeto respondiese de una forma adecuada.

En un segundo nivel, el estrés del conductor resultaría de las exposiciones continuadas al tráfico, las cuales exceden las habilidades del propio conductor para responder de forma segura y adecuada. Serian pequeñas situaciones de estrés, que se van acumulando diariamente en las respuestas emocionales, cognitivas y fisiológicas del sujeto.

Otra de las cuestiones relativas a la ansiedad en la conducción, y que ha sido ampliamente investigada, es la concerniente a la ansiedad como rasgo de la personalidad (Fairclough et al., 2006; Wilson, Smith, Chattington, Ford \& Marple-Horvat, 2006). Parece ser que los sujetos con rasgos de ansiedad más elevados son más proclives a tener accidentes. En este sentido, se ha demostrado que los sujetos con emociones más positivas son los conductores que se sienten más seguros a la hora de conducir, ya que disfrutan de su tarea (Alonso et al., 2006a).

El conductor ansioso se caracteriza por ser un individuo con alto nivel de ansiedad-rasgo, suele tener facilidad para el aprendizaje y un bajo nivel de impulsividad. Cuanto más ansioso es, mejor acepta el sistema de leyes de tráfico como legítimo y suele definirse como un sujeto que acepta y sobre todo cumple todo tipo de leyes, incluyendo las de tráfico. Su deseo de tomar riesgos es muy bajo debido precisamente al cumplimiento de la normativa vial. Sin embargo, en cualquier situación conflictiva 0 ambigua en la carretera toma mayores riesgos de tal forma que intenta con ello liberar la tensión. Este conductor ansioso confunde sus emociones con sus decisiones y llega a poseer un estado de ansiedad en aquellas situaciones donde se le exige una decisión rápida y casi instintiva. En general, tanto las presiones como las situaciones de tráfico le sitúan en un conflicto de acercamiento-evitación, parecido al modelo de Amenaza-Evitación de Fuller. Con todo ello, el sujeto procesará de forma incorrecta la información y, por lo tanto, tendrá bastantes posibilidades de cometer una infracción y en el peor de los casos podrá sufrir algún tipo de accidente.

\section{RESULTADOS}

Las habilidades emocionales, en general, suponen un buen pronóstico de las conductas de riesgos de los sujetos al volante. De tal forma que aquellos usuarios que utilizan más habilidades emocionales son los que más conductas seguras tienen en la conducción (Arnau-Sabatés, Sala-Roca \& Jariot-García, 2012).

En los resultados de la investigación, en relación con la Inteligencia Emocional en general, los conductores se sitúan cerca de la media en los tres componentes, pero son los conductores con menos experiencia los que tienen mejores niveles de atención de las emociones y menores niveles en el componente de comprensión, donde los profesionales alcanzan mayores puntuaciones.

La edad juega aquí un papel fundamental: son los más jóvenes los que están más atentos a las emociones de los demás. Esta habilidad corresponde al nivel más básico de la Inteligencia emocional y permite adaptarnos mejor a nuestro entorno (Extremera \& Fernández-Berrocal, 2004; León, 2004; Teruel, 2000; Van Der Zee, Thies \& Schakel, 2002).

En el caso de los profesionales, su madurez les permite tener una mejor comprensión de sus estados de ánimo, cuestión que es fundamental para una mejor conducción, dado que por lo general adaptan su humor a las situaciones de tráfico, permitiéndoles ser menos vulnerables ante cualquier circunstancia. Hay que recordar lo importante que es tener un autocontrol emocional para la seguridad, dado que el enfado y las reacciones agresivas del conductor son señales de la conducta infractora al volante (Dahlen et al., 2005; Delhome \& Villieux, 2005) 
Tabla 1 Atención, percepción y regulación emocional y grupo

\begin{tabular}{|c|c|c|c|c|}
\hline Grupo & & Atención & Claridad_ & Regulación \\
\hline \multirow{4}{*}{ Novel } & Media & 1,85 & 1,90 & 1,45 \\
\cline { 2 - 5 } & $\mathrm{N}$ & 160 & 160 & 160 \\
\cline { 2 - 5 } & Desv. típ. &, 581 &, 702 &, 512 \\
\hline \multirow{4}{*}{ Experto } & Media & 1,53 & 2,17 & 1,33 \\
\cline { 2 - 5 } & $\mathrm{N}$ & 153 & 153 & 153 \\
\cline { 2 - 5 } & Desv. típ. &, 573 &, 689 &, 475 \\
\hline \multirow{3}{*}{ Total } & Media & 1,69 & 2,03 & 1,39 \\
\cline { 2 - 5 } & $\mathrm{N}$ & 313 & 313 & 313 \\
\cline { 2 - 5 } & Desv. típ. &, 598 &, 708 &, 497 \\
\hline
\end{tabular}

\section{ANSIEDAD}

En nuestra investigación, los niveles de ansiedad de los conductores, en general, han sido normales y se sitúan en la media. Aunque son los conductores noveles los que han manifestado ciertos índices de alteración de la ansiedad, no así los profesionales que se muestran más tranquilos y con niveles más bajos (Ledesma et al., 2007; Saiz et al., 1997). Al igual que Fernández \& Duval (2010), se puede afirmar que los profesionales son menos ansiosos, debido principalmente a su rutina en el trabajo. La mayor experiencia en la conducción les proporciona mayores niveles de control sobre su estado de ansiedad, lo cual repercute en un comportamiento más seguro en las vías. Así lo constatan también Gwyther \& Holland (2011) en un estudio realizado con conductores noveles, adultos y mayores. En dicha investigación, se pone de manifiesto que los procesos de autorregulación en los conductores mejoran conforme lo hace el control de la ansiedad, de tal forma que la experiencia condiciona un mayor uso de la autorregulación en la conducción.

Tabla 1 Atención, percepción y regulación emocional y grupo

\begin{tabular}{|c|l|c|c|}
\hline \multicolumn{2}{|c|}{} & \multicolumn{2}{c|}{ Grupo } \\
\cline { 3 - 4 } \multicolumn{5}{|c|}{} & 1 & Novel & Experto \\
\cline { 2 - 4 } & 2 & $2,5 \%$ & $9,8 \%$ \\
\cline { 2 - 4 } & 3 & $11,9 \%$ & $17,0 \%$ \\
\cline { 2 - 4 } Estado & 4 & $13,8 \%$ & $17,6 \%$ \\
\cline { 2 - 4 } & 5 & $20,6 \%$ & $19,6 \%$ \\
\cline { 2 - 4 } & 6 & $18,8 \%$ & $10,5 \%$ \\
\cline { 2 - 4 } & 7 & $7,4 \%$ & $11,8 \%$ \\
\cline { 2 - 4 } & 8 &, $6 \%$ & $9,8 \%$ \\
\cline { 2 - 4 } & 9 & ---- & $3,3 \%$ \\
\hline
\end{tabular}




\section{EMOCIONES EN LA CONDUCCIÓN DE VEHÍCULOS: DIFERENCIAS ENTRE ESTUDIANTES Y PROFESIONALES}

Existe una serie de autores que sostienen que la mayor capacidad de control sobre la carretera está determinada por la experiencia en la conducción y no por la edad del conductor. Por lo tanto, es la falta de confianza la que eleva los niveles de ansiedad (Dahlen \& White, 2006; Horta, 2005). Como hemos comprobado en esta investigación, los conductores con mayor experiencia son los que tienen a su vez mayores niveles de seguridad en su conducción. Pero también es cierto que los resultados obtenidos demuestran que a mayor edad mayores niveles de seguridad, hecho que coincide con el estudio realizado por la $\operatorname{OCDE}^{1}(2006)$ sobre los jóvenes conductores, donde la variable edad tiene un importante papel en la conducción. A mayor edad, mayores niveles de maduración tanto física como mental y, por lo tanto, mayores niveles de seguridad (Dogan, Steg, Delhomme, \& Rothengatter, 2012)

\section{CONCLUSIONES}

En general, aquellos conductores que tienen niveles adecuados tanto de atención como de compresión de las propias emociones son los que más habilidades metacognitivas utilizan. Sobretodo, ha sido en la dimensión claridad o comprensión de las emociones donde se han encontrado un mayor número de diferencias significativas en las distintas habilidades, dimensión que, como se ha comentado, es la que poseen con mayor porcentaje los conductores profesionales. Un mejor conocimiento de las propias emociones supone un mayor uso de las habilidades y estrategias cognitivas, lo que conlleva un mejor autocontrol de las conductas y de los impulsos (Extremera \& Fernández-Berrocal, 2004; Teruel, 2009).

\section{REFERENCIAS BIBLIOGRÁFICAS}

Arnau-Sabatés, L., Sala-Roca, J. \& Jariot-García, M. (2012). Emotional abilities as predictors of risky driving behavior among a cohort of middle aged drivers. Accident; Accident Analysis and Prevention ,45, 818-825.

Alonso, F., Esteban, C., Calatayud, Alamar, B. \& Egido, A. (2006a). Emociones y conducción. Teoría y Fundamentos. Cuadernos de Reflexión Attitudes

Alonso, F., Sanmartín, J., Esteban, C., Calatayud, C., Alamar, B. \& López, E. (2006b). Emociones y conducción: Una visión a partir de los sentimientos de la población española. Cuadernos de Reflexión Attitudes.

Bisquerra, R. (2000). Educación emocional y bienestar. Barcelona: Praxis.

Cale, M. H. \& Shaked, E. (2004). The effects of fear of terrorist attacks on driving behavior and traffic safety. Israel: CogniTo Ltd.

Clapp, J. D., Olsen, S. A., Beck, J. G., Palyo, S. A., Grant, D. M., Gudmundsdottir, B. et al. (2011) The Driving Behavior Survey: Scale development and validation. Journal of Anxiety Disorders, $25,96-105$.

Dogan, E., Steg, L., Delhomme, P. \& Rothengatter, T. (2012). The effects of non-evaluative feedback on drivers' self-evaluation and performance. Accident Analysis and Prevention 45, 522-528.

León, Ch. (2004). La Inteligencia Emocional en Estudiantes de Educación Superior Universitaria y no Universitaria de Administración de Empresas. Recuperado el 11 de enero de 2019 en http://www.psiologiacientifica.com/pblicaciones/biblioteca/articulos/ar- porras02.html.

Ledesma, R., Peltzer R. \& Poó, F. (2006). Evaluación de estilos de conducción. 1ra Jornada Regional de Psicología del Tránsito y Accidentología. Colegio Oficial de Psicólogos, Dist. X, Mar del Plata, 2 de diciembre de 2006.

Levelt, P. B. M. (2003). Field study of emotions in traffic; Questionnaire study of features such as frequency, cause and road safety effects. Swov.

Stephens, A. N. \& Groeger, J. A. (2009). Situational specificity of trait influences on drivers' evalua- 
tions and driving behaviour. Transportation Research Part F: Traffic Psychology and Behaviour, 12, 29-39

Swov (2003). Emotions in Traffic. Swov Research Activities 23 September 2003.

- (2005). Emotions and Traffic. Fact Sheet. Institute for Road Safety Research. The Netherladns. 28 April, 2005.

1 La organización para la cooperación y el desarrollo económico. Los Países-miembros de la OCDE son: Australia, Austria, Bélgica, Canadá, República Checa, Dinamarca, Finlandia, Francia, Alemania, Grecia, Hungría, Islandia, Irlanda, Italia, Japón, Corea, Luxemburgo, México, Holanda, Nueva Zelanda, Noruega, Polonia, Portugal, República Eslovaca, España, Suecia, Suiza, Turquía, el Reino Unido y los Estados Unidos. La Comisión de la Comunidad Europea también está involucrada en el trabajo de la OCDE. 
